\title{
Salt intakes of rural and urban Gambian women
}

\author{
S.E. Dalzell ${ }^{1}$, L.M.A. Jarjou ${ }^{2}$, A. Prentice ${ }^{1,2}$, K. Ward ${ }^{1}$ and G.R. Goldberg ${ }^{1,2}$ \\ ${ }^{1}$ Medical Research Council Elsie Widdowson Laboratory, Cambridge CB1 9NL, UK and ${ }^{2}$ Medical Research Council \\ Unit The Gambia at the London School of Hygiene and Tropical Medicine, The Gambia.
}

Sodium is an essential nutrient and salt is the major source of sodium in the diet. In many high-income countries (HIC), dietary salt comes primarily from processed food rather than an addition to food in the home. Although intakes of 'processed food' are increasing in low and middle income countries (LMIC) like The Gambia, they are still low compared to many HIC, and high salt intake is not yet prioritised as a major health issue in many LMICs.

Whilst the use of local and commercially produced salt is traditional and widespread, more recent increases in availability and use of stock cubes, monosodium glutamate, and bottled and packet sauces suggest that salt intakes may be increasing. There is also evidence that these are used additively rather than singly and thus could increase sodium intakes significantly (Gambian National Nutrition Agency, personal communication). Locally baked bread also contains significant amounts of salt; a key characteristic of the nutrition transition is an increase in bread consumption ${ }^{(1)}$. A systematic review in sub Saharan Africa showed that most countries have salt intakes exceeding recommendations, with urban populations more likely to have higher intakes than rural ${ }^{(2)}$.

Measuring sodium excretion from 24-hour urine collections is the most accurate way to assess daily salt intake. Dietary intake methods are not considered as reliable, since salt added during cooking or during a meal is not always taken into account, and because of measurement errors associated with these methods. Salt cannot be stored in the body and has to be excreted by the kidneys. As part of this physiological process, there is obligatory excretion of calcium. Hence, high sodium intakes as well as increasing risk of cardiovascular disease are adverse for bone health, associated with higher bone resorption and increased calciuria ${ }^{(3,4)}$.

We have conducted a study investigating the impact of rural-to-urban migration on bone health in Gambian women ${ }^{(5,6)}$. We present here results for sodium/salt in two groups of pre-menopausal women: urban migrant $(n=58)$ and rural ( $n=71)$. 24-hour urine samples were collected and aliquots analysed for sodium and for calcium using ion-specific electrodes on the Siemens Dimension ${ }^{\circledR}$ Xpand clinical chemistry system with the QuikLYTE ${ }^{\circledR}$ module.

Sodium excretion was similar in both groups, rural 98.0 (IQR: 62.6 to 133.5$) \mathrm{mmol} / 24 \mathrm{~h}$ and urban 112.3 (IQR: 72.4 to $145 \cdot 7$ ) $\mathrm{mmol} / 24 \mathrm{~h}(p>0.05)$. Sodium excretion expressed as salt equivalent $(17 \cdot 1 \mathrm{mmol} \mathrm{Na}=1 \mathrm{~g}$ salt $)$, showed median intakes to be, rural 5.7 (IQR: 3.7 to 7.8 ) g/day and urban 6.5 (IQR: 4.2 to 8.5 ) g/day. Intakes ranged from 2.0 to $15.4 \mathrm{~g}$, and 1.8 to $12.4 \mathrm{~g}$ in rural and urban women respectively. As a proportion, $72 \%$ and $78 \%$ of rural and urban women had salt intakes above $4 \mathrm{~g}$, while $42 \%$ of rural and $55 \%$ of urban, had intakes above $6 \mathrm{~g}$. These values are consistent with the ingredients and recipe data collected as part of our dietary intake measurements in this study ${ }^{(6)}$. Urinary calcium excretion was positively associated with increasing sodium excretion $(p<0 \cdot 01)$. Further analysis is underway to investigate sodium and potassium intakes in relation to bone phenotype and markers of bone turnover.

These data indicate that salt intakes in both rural and urban Gambian women are high compared to recommendations (e.g. maximum of $5 \mathrm{~g} /$ day recommended by the WHO, the UK reference nutrient intake of $4 \mathrm{~g} / \mathrm{day}$, and maximum of $6 \mathrm{~g} / \mathrm{day}$ ). Larger studies with nationally representative samples are needed to understand the prevalence and distribution of high salt intakes in The Gambia and effects on health.

Supported by the UK Medical Research Council (MRC) under programmes U105960371 and U123261351. This research is jointly funded by the MRC and the Department for International Development (DFID) under the MRC/DFID Concordat agreement. SD is in receipt of an MRC PhD studentship.

1. Sodjinou R, Agueh V, Fayomi B et al. (2007) European Journal of Clinical Nutrition 63, 222-228.

2. Oyebode O, Oti S, Chen Y-F et al. (2016) Population Health Metrics 14, 1.

3. Prentice A (2004) Public Health Nutr 7, 227-243.

4. Teucher B, Dainty JR, Spinks CA et al. (2008) J Bone Miner Res 23, 1477-1485.

5. Dalzell SE, Jarjou LMA, Prentice A et al. (2017) Proceedings of the Nutrition Society 76.

6. Dalzell SE, Jarjou LMA, Prentice A et al. (2017) Proceedings of the Nutrition Society 76. 